\title{
SOME PROPERTIES OF MEAN PERIODIC FUNCTIONS
}

\author{
P. G. LAIRD \\ (Received 12 April 1970) \\ Communicated by J. B. Miller
}

This paper presents some properties of continuous complex-valued mean periodic functions of a single real variable. The theory of these functions is due mainly to Schwartz [7] and they have also been considered by Kahane $[4,5]$. Most of the properties outlined here are also shared by the continuous meanperiodic functions on a half line introduced by Koosis [6].

We use $C(R)$ to denote the set of all continuous complex-valued functions defined on the real line, $R$, taken with the topology of convergence uniform on all compact subsets of $R$. A function $f$ belonging to $C(R)$ is mean periodic if the linear subspace spanned by $f$ and its translates is not dense in $C(R)$; this condition is equivalent to each of: (i) the existence of a non-zero measure, $\mu$, of compact support such that $\mu * f=0$, i.e.

$$
\int f(t-r) d \mu(r)=0 \text { for all real } t
$$

(ii) $f$ is the limit in $C(R)$ of a sequence of exponential polynomials $f_{n}$, each satisfying $\mu * f_{n}=0$ for some non-zero measure $\mu$ of compact support. Here by an exponential polynomial is meant a finite linear combination of terms $u_{n} e_{a}$ where $u_{n} e_{a}: t \rightarrow t^{n} e^{a t}, n$ is any non-negative integer and $a$ is any complex number.

Throughout this note, we will understand the term 'measure' to mean a measure of compact support and use the facts that the convolution of two measures, is a measure and that operation of convolution of measures with measures or measures with continuous functions restricted to a half line, is associative and commutative (see, for example, Edwards [2], 4.19). Also, $f$ is used to denote a mean periodic function belonging to $C(R)$ and $\mu$ a non-zero measure such that $\mu * f=0$. From this characterization, it follows that any primitive of $f$ or the derivative of $f$ (when it is continuous) is mean periodic and both have the same mean period of $f$. The mean period of $f$ is the infimum of the lengths of the intervals containing the supports of the non-zero measures $\mu$ such that $\mu * f=0$.

We note that $\mu * \lambda$ is a non-zero measure when $\mu$ and $\lambda$ are non-zero measures. 
Also $\mu * \lambda$ has support contained in an interval of length $2 \varepsilon$ when the lengths of the supporting intervals of $\mu$ and $\lambda$ do not exceed $\varepsilon$. If $M P$ denotes the set of all mean periodic functions in $C(R)$ and $M P_{0}$ the set of mean periodic functions with mean period zero, then it follows that $M P$ and $M P_{0}$ are linear subspaces of $C(R)$. The set of exponential polynomials is a linear subspace of $M P_{0}$ and the set $M P$ is a proper dense subspace of $C(R)$.

The truncated convolution product of two $C(R)$ functions $x$ and $y$ is defined by

$$
x \circledast y: t \rightarrow \int_{0}^{t} x(t-r) y(r) d r
$$

and $x \circledast y \in C(R)$. With addition and this product, $C(R)$ is an algebra (see Erdélyi [3] for this and other details of this product).

Proposition 1. The truncated convolution product of two mean periodic functions is mean periodic.

Proof. Let $f, g \in M P$ and, as well as $\mu * f=0$, suppose that $\lambda$ is a nonzero measure such that $\lambda * g=0$. Let $f^{-}$be equal to $f$ on $(-\infty, 0)$ and be zero otherwise; also let $f^{+}=f-f^{-}$and define $g^{-}$and $g^{+}$in the same way. Then $v=\mu * f^{-}$is a function of compact support and as

$$
\begin{aligned}
f^{+} * g^{+} & =(f \circledast g)^{+} \text {and } f^{-} * g^{-}=-(f \circledast g)^{-}, \text {then } \\
\mu *(f \circledast g) & =\mu *\left(f^{+} * g^{+}-f^{-} * g^{-}\right) \\
& =(-v) * g^{+}-v * g^{-} \\
& =-v * g .
\end{aligned}
$$

Hence $\mu * \lambda *(f \circledast g)=-v * g * \lambda=0$ and as $\mu * \lambda$ is a non-zero measure, $f \circledast g$ is mean periodic.

COROLlary. $M P_{0}$ and $M P$ are subalgebras of $C(R)$.

Proposition 2. If $f$ and $f \circledast g$ are mean periodic, if $f$ is non-zero and if $g$ is continuous, then $g$ is mean periodic.

Proof. As above, set $v=\mu * f^{-}$and note that as well as $v$ having compact support, it is non-zero for $f$ and hence $f^{-}$are non-zero. Let $v$ be a non-zero measure such that $v *(f \circledast g)=0$; then from $\mu *(f \circledast g)=-v * g$, it follows that $v * v * g=0$ and as $v * v$ is a non-zero measure, $g$ is mean periodic.

COROllaRY. Let $f$ be non-zero and $g$ be continuous. If $f$ and $f \circledast g$ have mean period zero then $g$ has mean period zero. 
PROPOSITION 3. If $f$ is mean periodic and if $g$ is an exponential polynomial, then $f g$ is mean periodic.

Proof. We firstly consider $u f$ where $u f: t \rightarrow t f(t)$. With $\mu * f=0$,

$$
\mu * u f=u(\mu * f)-\lambda * f=-\lambda * f
$$

where $\lambda$ is the non-zero measure defined by $\lambda(x)=\mu(u x)$ for each $x \in C(R)$. Hence

$$
\mu * \mu * u f=-\mu * \lambda * f=0
$$

and as $\mu * \mu$ is a non-zero measure, $u f$ is mean periodic. Thus $u_{n} f$ is mean periodic. Since multiplication by an exponential does not affect a function's mean periodicity, $u_{n} e_{a} f$ is mean periodic. Hence $f g$ is mean periodic.

COROLlary. Let $g$ be an exponential polynomial. Then $f g$ is mean periodic with mean period zero when $f$ is.

The following examples show that the pointwise product of two mean periodic functions need not be mean periodic: let $f$ and $g$ be two continuous periodic functions that have no non-zero Fourier coefficients $\hat{f}(m), \hat{g}(n)$ ( $m, n$ any integers) where $f$ has period $2 \pi / \alpha, g$ has period $2 \pi / \beta$ and $\alpha / \beta$ is irrational.

To show that $f g$ is not mean periodic, we suppose that $\lambda$ is any measure with a compact support $K$ satisfying $\lambda *(f g)=0$ and let $\sigma_{N} f$ and $\sigma_{N} g$ denote the Cesàro means associated with $f$ and $g$ so that $\sigma_{N} f \times \sigma_{N} g$ converges uniformly to $f g$ as $N \rightarrow \infty$. Using the fact that

$$
\frac{1}{T} \int_{-r}^{T-r} e^{i \theta s} d s
$$

converges uniformly in $r$ to 0 as $T \rightarrow \infty$ if $\theta \neq 0$ and

it follows that

$$
\frac{1}{T} \int_{-r}^{T-r} d s=1
$$

$$
\begin{gathered}
\lim _{T \rightarrow \infty} \frac{1}{T} \int_{-r}^{T-r} f(t) g(t) e^{-i \gamma t} d t \\
=\lim _{N \rightarrow \infty} \sum_{|m| \leqq N} \sum_{|n| \leqq N}\left[1-\frac{|m|}{N+1}\right]\left[1-\frac{|n|}{N+1}\right] \\
\times \hat{f}(m) \hat{g}(n) \lim _{T \rightarrow \infty} \frac{1}{T} \int_{-r}^{T-r} e^{i \alpha m t+i \beta n t-i \gamma t} d t \\
= \begin{cases}\hat{f}(m) \hat{g}(n) & (\gamma=\alpha m+\beta n), \\
0 & \text { (otherwise). }\end{cases}
\end{gathered}
$$


Hence when $\gamma=\alpha m+\beta n$,

$$
\begin{aligned}
\hat{f}(m) \hat{g}(n) \int_{K} e^{-i \gamma r} d \lambda(r) & =\lim _{T \rightarrow \infty} \int_{K} e^{-i \gamma r} d \lambda(r) \frac{1}{T} \int_{-r}^{T-r} f(s) g(s) e^{-i \gamma s} d s \\
& =\lim _{T \rightarrow \infty} \frac{1}{T} \int_{0}^{T} e^{-i \gamma t} \int_{K} f(t-r) g(t-r) d \lambda(r) d t \\
& =0 .
\end{aligned}
$$

$$
M(z)=\int_{K} e^{-i z t} d \lambda(t)
$$

the Fourier transform of the measure $\lambda$, we see that $M$ vanishes on the set $S=\{\alpha m+\beta n: m, n$ are integers $\}$. As $M$ is an entire function and $S$ is dense in the real line, it follows that $M$ is zero. Hence $\lambda$ is zero and so $f g$ cannot be a mean periodic function.

In the above example, $f$ and $g$ have mean periods of $2 \pi / \alpha$ and $2 \pi / \beta$. If we now have $\hat{f}(m)$ non-zero when $m= \pm p^{2}$ and zero otherwise and $\hat{g}(n)$ non-zero when $n= \pm q^{2}$ and zero otherwise ( $p, q$ integers), then both $f$ and $g$ have mean period zero since the spectrum $\left\{a_{k}\right\}$ of either function is real and simple and satisfies $\Sigma 1 /\left|a_{k}\right|<\infty$.

From the above calculations, if $v$ is any measure satisfying $v *(f g)=0$ and if $y$ has Fourier transform $L$, then $L$ vanishes on the set $S^{\prime}=\left\{ \pm \alpha p^{2} \pm \beta q^{2}: p, q\right.$ are integers $\}$. When $\alpha / \beta$ is irrational and $\sqrt{\alpha / \beta}$ is approximable by rationals to order three, then for any positive $\varepsilon$, an infinity of integers $p, q$ can be found such that $\left|\alpha p^{2}-\beta q^{2}\right|<\varepsilon$. Thus $S^{\prime}$ has zero as a limit point and as $L$ is an entire function, it must be zero. Thus $v$ is zero and so $f g$ is not mean periodic.

We now consider the relationship between mean periodic functions and linear differential equations with constant coefficients and other functional equations.

PROPOSITION 4. The differential equation $x^{\prime}+a x=y$ where $a$ is a constant, $y \in C(R)$ and $x(\alpha)=c$ has a mean periodic solution if and only if the forcing term $y$ is mean periodic.

Proof. It is trivial that $y$ is mean periodic when $x$ is mean periodic. Conversely, the solution of this equation is

$$
x(t)=e^{-a t}\left[c e^{a \alpha}-\int_{0}^{\alpha} e^{a r} y(r) d r\right]+e_{-a} \circledast y(\mathrm{t})
$$

which, by Proposition 1, is mean periodic when $y$ is.

COROLlary. When the solution and forcing term are mean periodic, both have the same mean period. 
Proposition 5. For the system of equations,

$$
x^{\prime}(t)+A x(t)=y(t) \text { with } x(\alpha)=c
$$

where $y$ is an n-vector valued function with continuous components and $A$ is $a$ constant $n \times n$ matrix, a necessary and sufficient condition that $x$ be mean periodic is that $y$ be mean periodic.

Proof. If $x$ is mean periodic in the sense that there exists a non-zero measure $\lambda$ such that $\lambda * x_{1}=0$ for each component of $x$, then as $x$ is continuously differentiable, $\lambda * x^{\prime}=0$; also $\lambda * A x=A \lambda * x=0$ so that $\lambda * y=0$ showing $y$ is mean periodic.

Conversely, if $y$ is mean periodic and such that $v * y=0$ where $v$ is a non-zero measure, set $z=v * x$ so that

$$
z^{\prime}+A z=v * x^{\prime}+A v * x=v *\left(x^{\prime}+A x\right)=v * y=0 .
$$

Then $z(t)=e^{-A t} d$ where $d$ is some constant vector. As the elements of the matrix $e^{-A t}$ are exponential polynomials, the components of $z$ are exponential polynomials. Thus $\psi * z=0$ and so $\psi * v * x=0$ where $\psi$ and $\psi * v$ are non-zero measures, and so $x$ is mean periodic.

Proposition 6. For the two kinds of integral equations of convolution type

$$
\begin{aligned}
x \circledast f & =y \\
x-a x \circledast f & =y
\end{aligned}
$$

where $a$ is a non-zero constant, $y$ is continuous and $f$ is a non-zero mean periodic function, if $x$ is mean periodic, then so is $y$. Conversely, if $y$ is mean periodic, there is only one continuous solution and this is mean periodic.

Proof. The first statement follows directly from Proposition 1. For the converse part of (1), the mean periodicity of any continuous solution is due to Proposition 2 and its uniqueness is due to the fact that the difference between any two continuous solutions, say $z$, is mean periodic and satisfies $z \circledast f=0$. As a non-zero mean periodic function cannot vanish on a half line, $f^{+}$and $f^{-}$are non-zero so from Titchmarch's convolution theorem, $z^{+}$and $z^{-}$are zero so that $z$ is zero.

For (2) when $y$ is mean periodic with $\lambda * y=0$ where $\lambda$ is a non-zero measure and $\mu * f^{-}=v$,

$$
\mu * y=\mu * x+a x * v
$$

so that $\lambda *(\mu+a v) * x=0$. As $\mu+a v=\mu *\left(\delta+a f^{-}\right)$is a non-zero measure, it follows that $x$ is mean periodic when it is continuous. If $z$ is the difference between any two continuous solutions of (2), then 


$$
z=a(z \circledast f)=z \circledast(a f)
$$

and since $a f$ is continuous, $a f \neq \delta$ and so $z=0$. Here, $\delta$ denotes the Dirac measure with $\delta(g)=g(0)$ and $\delta * g=g$ for each $g \in C(R)$.

Proposition 7. For the differential-difference equation

$$
x^{\prime}(t)+\sum_{k=0}^{n} a_{k} x\left(t-\omega_{k}\right)=y(t)
$$

where $y$ is continuous on $R, n>0, a_{0}, a_{1}, \ldots, a_{n}$ are non-zero complex numbers and $\omega_{0}<\omega_{1}<\ldots<\omega_{n}$ are real numbers, if any solution is mean periodic, then $y$ is mean periodic. Conversely, if $y$ is mean periodic, then all solutions valid on $R$ are mean periodic.

Proof. Any solution of (3) that is valid on the real line is continuously differentiable so that if it is mean periodic as well, $x^{\prime}$ together with the translates of $x$ are mean periodic. Thus $y$ is mean periodic.

Conversely, let $T$ denote the distribution $D \delta+\sum_{k=0}^{n} a_{k} \delta_{\omega_{k}}$ where $\delta_{\omega}$ is the Dirac measure placed at $\omega$ so that (3) can be written as $T * x=y$. Since $y$ is mean periodic, there exists a non-zero measure $\lambda$ such that $\lambda * y=0$. If $\rho$ is any non-zero indefinitely differentiable function with a compact support, then so is $\psi=\rho * \lambda * T$ and $\psi * x=0$. Thus $x$ is mean periodic.

We note that the above propositions and their corollaries are valid for the continuous complex-valued mean periodic functions defined on $R_{+}$, the set of nonnegative real numbers (Koosis [6]). The proofs in the case of these functions resemble those given above and are omitted. However, Proposition 7 can be modified to give

Proposition 8. For the differential-difference equation (3) of retarded type where

(i) $\quad y$ is continuous on $R_{+}$

(ii) $0=\omega_{0}<\omega_{1}<\ldots<\omega_{n}=\alpha, n>0, a_{n} \neq 0$,

(iii) $g$ is continuous on $[0, \alpha]$,

(a) there exists a unique function, $x$ which is continuous on $R_{+}$, satisfies (3) on $(\alpha, \infty)$ and coincides with $g$ on $[0, \alpha]$. Moreover, $u$ is continuously differentiable on $(\alpha, \infty)$, and

(b) a necessary and sufficient condition that the solution be mean periodic on a half line is that $y$ be mean periodic on a half line.

Proof. The proof of (a) is routine. For (b), there is no restriction in assuming that $x$ and $y$ are defined on $R$ and zero on $(-\infty, 0)$. Equation (3) can be written as 


$$
x^{\prime}(t+\alpha)+\sum_{k=0}^{n} a_{k} x\left(t+\alpha-\omega_{k}\right)=y(t+\alpha) \text { for } t>0 .
$$

When the solution is mean periodic on a half line since it is also continuously differentiable on $(\alpha, \infty)$, the function $T_{-\alpha} x^{\prime}: t \rightarrow x^{\prime}(t+\alpha)$ along with the translates of $x$ and so $T_{-\alpha} y$ are mean periodic on a half line. Thus there exists a non-zero measure $\lambda$ with support contained in $[-\beta, 0]$ for some $\beta>0$ such that $\lambda * T_{-\alpha} y=\delta_{-\alpha} * \lambda * y$ is zero on $(0, \infty)$. As $\delta_{-\alpha} * \lambda$ is a non-zero measure with a compact support in $(-\infty, 0], y$ is mean periodic on a half line.

Equation (4) can be written as $S * x=T_{-\alpha} y$ where $S$ is the distribution

$$
D \delta_{-\alpha}+\sum_{k=0}^{n} \alpha_{k} \delta_{-\alpha+\omega_{k}}
$$

with support contained in $[-\alpha, 0]$. When $y$ is mean periodic on a half line with $v$, a non-zero measure such that $v$ and $v * y$ have compact supports in $(-\infty, 0]$, then $\nu * T_{-\alpha} y=\nu * S * x$ also has compact support in $(-\infty, 0]$. When $\rho$ is any non-zero indefinitely differentiable function with a compact support in $(-\infty, 0]$, so also is $\psi=\rho * v * S$. Thus $\psi * x$ is zero on $(0, \infty)$ so that $x$ is mean periodic on a half line.

NOTE. An alternative method of proving the converse portion of the above (and also Proposition 7) that is longer and more constructive is now outlined. The characteristic equation of (3) is

$$
h(z)=z+\sum_{k=0}^{n} a_{k} \exp \left(-z \omega_{k}\right)=0
$$

and has at least one root, $z=c$ say. For if not, $h(z)$ is of the form $e^{g(z)}$ where $g(z)$ is an entire function. As $h(z)$ is of order one, $g(z)=d z+A$ where $A$ and $d$ are constants so that $h(z)=e^{A} e^{d z}$ which is contradictory.

Now set $x(t)=e^{c t} v(t)$ so that (4) becomes

$$
v^{\prime}(t+\alpha)-\sum_{k=0}^{n} a_{k} \exp \left(-c \omega_{k}\right)\left(v(t+\alpha)-v\left(t+\alpha-\omega_{k}\right)\right)=e^{-c t-c \alpha} y(t+\alpha) .
$$

On integration and rearrangement this equation gives

$$
v(t+\alpha)-\sum_{k=0}^{n} a_{k} \exp \left(-c \omega_{k}\right) \int_{-\alpha}^{-\alpha+\omega_{k}} v(t-r) d r=A+F(t)
$$

where

$$
A=v(\alpha)-\sum_{k=0}^{n} a_{k} \exp \left(-c \omega_{k}\right) \int_{0}^{\omega_{k}} v\left(r+\alpha-\omega_{k}\right) d r
$$


a constant that may be determined from the boundary condition and

$$
F(t)=\int_{\alpha}^{\alpha+t} e^{-c r} y(r) d r .
$$

If $\lambda$ is the measure with support $[-\alpha, 0]$ defined by

$$
\lambda(w)=w(-\alpha)-\sum_{k=0}^{n} a_{k} \exp \left(-c \omega_{k}\right) \int_{-\alpha+\omega_{k}}^{-\alpha} w(r) d r
$$

for each continuous function $w$, then (5) can be written as $\lambda * v=A+F$. When $y$ is mean periodic on a half line, so also are $F$ and $A+F$ so there exists a non-zero measure $v$ such that $v$ and $v *(A+F)$ have compact supports in $(-\infty, 0]$. Hence $v * \lambda * v$ and the non-zero measure $v * \lambda$ have compact supports in $(-\infty, 0]$ showing that $v$ and $x$ are mean periodic on a half line.

COROLlaRY. When $y$ is zero, the solution $x$ is uniformly approximable on all compact subintervals of $(\alpha, \infty)$ by finite linear combinations of exponential monomials $u_{n} e_{a}$ where the characteristic equation $h(z)=0$ has a zero of order exceeding $n$ at $z=a$.

Proof. That the mean period of the solution does not exceed $\alpha$ follows from $\rho * S * x=0$ on $(0, \infty)$ when $y=0$ since $\rho$ can be chosen with support contained in an interval of arbitrarily small length and $S$ has support $[-\alpha, 0]$. The spectrum of $x, \Lambda_{x}$, is contained in the set $\{(\alpha, p): S(z)$ has a zero of order $p$ at $z=a\}$ where $\hat{S}(z)$ is the Fourier transform of the distribution $S$ and equal to $S\left(e_{-i z}\right)$. Hence $x$ is uniformly approximable on compact subsets of $(\alpha, \infty)$ by linear combinations of exponential monomials $u_{n} e_{i a}$ where $(a, p) \in \Lambda_{x}$ and $n<p$ (Koosis [6]). As $S(z)=e^{i z a} h(i z)$, the statement of the corollary is proved.

This result forms part of the statement of Theorem 4.2 (Bellman and Cooke [1], page 109). However, our treatment using mean periodic functions does not readily give a series representation in terms of the exponential monomials or consider conditions when this series is uniformly convergent as Bellman and Cooke's approach does.

\section{Acknowledgements}

Most of this work formed part of an M.Sc. thesis presented to the Australian National University in 1969. My thanks are due to this university for providing a Research Scholarship, my supervisor, Dr. R. E. Edwards for suggesting the use of distributions with differential-difference equations and to Mr.W. A. Coppel for suggesting most of the problems. 


\section{References}

[1] R. Bellman and K. L. Cooke, Differential-Difference Equations (Academic Press, 1963).

[2] R. E. Edwards, Functional Analysis, Theory and Applications (Holt, Rinehart and Winston, 1965).

[3] A. Erdélyi, Operational Calculus and Generalized Functions (Holt, Rinehart and Winston, 1962).

[4] J. P. Kahane, 'Sur quelques problèmes d'unicité et de prolongement, relatifs aux fonctions approchables par des sommes d'exponentielles', Annales d'Institute Fourier, 5 (1953-54), 39-130.

[5] J. P. Kahane, Lectures on Mean Periodic Functions (Tata Institute of Fundamental Research, Bombay, 1959).

[6] P. Koosis, 'On functions which are mean periodic on a half line', Comm. Pure Appl. Math. 10 (1957), 133-149.

[7] L. Schwartz, 'Théorie générale des fonctions moyenne-périodiques', Annals of Math. 48 (1947), 857-929.

University of Otago

Dunedin, New Zealand

Present address

University of Calgary

Calgary, Alberta, Canada 